\title{
Cloning of fliC Gene From Salmonella typhimurium in the Expression Vector pVAX1 and Evaluation of its Expression in Eukaryotic Cells
}

\author{
Reza Taherkhani ${ }^{1}$; Fatemeh Farshadpour ${ }^{1}$; Manoochehr Makvandi ${ }^{1,}$; Ali Reza \\ Samarbafzadeh ${ }^{1}$ \\ ${ }^{1}$ Health Research Institute, Infectious and Tropical Disease Research Center, Ahvaz Jundishapur University of Medical Science, Ahvaz, IR Iran \\ ${ }^{*}$ Corresponding author: Manoochehr Makvandi, Health Research Institute, Infectious and Tropical Disease Research Center, Ahvaz Jundishapur University of Medical Sciences, Ah- \\ vaz, IR Iran. Tel: +98-9166181683, Fax: +98-6113738313, E-mail: manoochehrmakvandi29@yahoo.com
}

Received: May 20, 2013; Revised: September 22, 2013; Accepted: October 5, 2013

\begin{abstract}
Background: Flagellin is the main structural protein of the flagella of many pathogens including Salmonella typhimurium. It is a potent trigger of innate immune responses that enhance adaptive immune responses to a variety of protein antigens. Flagellin has intrinsic adjuvant activity mediated through toll-like receptor(TLR) 5 and is an attractive candidate for highly effective vaccine adjuvant conferring enhanced antibody and cellular immune responses to proteins or peptides. In the present study, we cloned the fliC gene from S. enterica typhimurium in eukaryote vector pVAX1 and evaluated its expression in eukaryotic cells.

Objectives: The main aim of the present study was to construct a DNA vaccine expressing fliC as an adjuvant.

Materials and Methods: The fliC gene of S. typhimurium (ATCC 14028) was amplified by PCR with specific primers and cloned into the pPrime cloning vector and successfully subcloned into expression vector pVAX1. The recombinant plasmid pVAX-fliC was finally expressed in eukaryotic cells.

Results: Cloning and subcloning of the fliC gene were confirmed by colony PCR, restriction enzymes digestion and DNA sequencing of the recombinant plasmids pPrime-fliC and pVAX-fliC. The expression of flagellin protein in eukaryotic cells was approved by immunofluorescence assay (IFA), western blotting analysis and the reverse transcriptase polymerase chain reaction (RT-PCR) method. Conclusions: The results of this study demonstrated that the fliC gene in recombinant plasmid pVAX-fliC was successfully expressed in eukaryotic cells and produced flagellin protein, which could be used as an effective adjuvant for DNA vaccine research.
\end{abstract}

Keywords:Cloning; fliC gene; pVAX1; Salmonella typhimurium; Adjuvant candidate

\section{Background}

Microorganisms such as bacteria and viruses have several components called pathogen-associated molecular patterns (PAMPs). Pattern recognition receptors (PRRs) of host innate immune system such as TLRs can recognize these components during infections $(1,2)$. Toll-like receptor proteins are members of a big family, which have extracellular domains with leucine-rich repeat (LRR), a transmembrane domain, and a intracytoplasmic region called the Toll/Interleukin-1 receptor (IL-1R) homology (TIR) domain $(1,2)$. At least 10 members of this family have been identified in mammalian cells, which can recognize specific components conserved among microorganisms $(1,3)$.

Toll-like receptor proteins are recognized by their ligands. Most of these ligands are found in the pathogens. Therefore, TLR proteins can act as primary stimulators of the innate immune system at the start of microbial infections $(1,3,4)$. Pathogen recognition by TLRs stimulates rapid activation of innate immunity by producing proinflammatory cytokines, chemokines, anti-microbial small molecules such as nitric oxide and upregulation of co-stimulatory molecules. Activated innate immunity subsequently leads to the development of effective antigen-specific adaptive immunity (1-3). Therefore, the use of TLR-ligands in vaccines could result in more potent and efficacious vaccines that can activate innate and adaptive immunity (5). Therefore the TLRs are considered to be adjuvant receptors $(1,6)$.

Flagellin, the major protein constituent of bacteria flagella from both Gram-positive and Gram-negative bacteria, is a mammalian TLR5 agonist. The activation of epithelial cells in response to Salmonella identified flagellin as a stimulatory ligand for TLR5 (7). TLR5 is expressed in epithelial cells, endothelial cells, macrophages, immature DCs, and T cells (2, 8-10).

Both innate and adaptive immunity can be activated by flagellin (11). Numerous studies have demonstrated the effectiveness of flagellin as a systemic and mucosal adjuvant to generate antigen-specific antibodies and to stimulate $\mathrm{T}$ cells in mice and nonhuman primates when it is administered either as a native purified protein or as a hybrid protein with physical linkage to target antigens 
and poorly immunogenic peptides (5, 12-17). Besides, systemic administration of flagellin may be a relatively safe means of providing temporary non-specific protection against a variety of challenges (18).

With this description, DNA encoding the TLR5 agonist (flagellin) as a potent adjuvant, with the ability to effectively stimulate the immune system, can be used either admixed or genetically linked to target antigens, particularly in studies of DNA vaccines. In a recent study, we generated a pVAX-fliC recombinant plasmid consisting of Salmonella enterica typhimurium flagellin, which is a TLR5 ligand and can be used as a genetic adjuvant candidate in eukaryote vector pVAX1 and we evaluated its expression in eukaryotic cells.

\section{Objectives}

In the present study, the fliC gene of S. enterica subsp. enteric serovar typhimurium ATCC 14028, encoding flagellin protein, was cloned into a the pPrime cloning vector and sub-cloned into a pVAX1 expression vector, and transfected into Hela cells, HEK293 cells and Chinese hamster ovary (CHO) eukaryotic cells. The main goal of this study was to construct the recombinant plasmid pVAX-fliC as an adjuvant candidate for DNA vaccines and evaluate its expression in eukaryotic cells.

\section{Materials and Methods}

\subsection{Bacterial Strains, Plasmid, Media, and Culture Conditions}

S. enterica subsp. enteric serovar typhimurium (ATCC 14028) was obtained from the American Type Culture Collection (ATCC, Manassas, VA., USA) and cultured on Salmonella Shigella agar medium (Oxoid CM0099, UK ) and Neutrient broth (Himedia, India) at $37^{\circ} \mathrm{C}$ and $5 \%$ $\mathrm{CO}_{2}$. Escherichia coli strain DH5 $\alpha$ (Novagen Inc., Madison, Wis., USA) was used for transformation and the pPrimecloning vector (5PRIME Inc. Germany) was used for cloning the PCR products, while pVAX1 (Invitrogen $₫$, Carlsbad, CA, USA) was used for sub-cloning the fliC gene. Eukaryotic expression vector pVAX1 carries the human cytomegalovirus (CMV) immediate early promoter, the bovine growth hormone (BGH) polyadenylation signal for transcription termination, the kanamycin resistance gene and the pUC origin of replication for maintenance in E. coli. The vector was constructed to be consistent with the Food and Drug Administration (FDA) document.

\subsection{Genomic DNA Extraction}

Genomic DNA of Salmonella enterica was extracted by High Pure PCR Template Preparation Kit (Roche, Germany), according to the manufacturer's instruction. Concentration and quality of the genomic DNA were assessed by UV absorbance and electrophoresis on $1 \%$ agarose gel.

\subsection{PCR Reaction}

PCR reaction was performed in a total volume of 50 $\mu \mathrm{l}$ containing $10 \mathrm{ng}$ of DNA, $20 \mathrm{pmol}$ of each primer, $200 \mu \mathrm{M}$ dNTP, $5 \mu \mathrm{l}$ of 10X PCR buffer, $1.5 \mathrm{mM} \mathrm{MgCl}_{2}$ and 2.5 U Expand High Fidelity PCR System. A pair of oligonucleotide primers were designed based on fliC DNA sequence that was recorded in GenBank (Accession number NC_016856, Region: 2059063-2060550). Forward primer: 5'-AA GCTAGCGGATCCACCATGGCACAAGTCATTAATACA-3' containing NheI and BamHI restriction sites with a simple Kozak sequence (CCACCATGG) just before the start codon to ensure proper translation of prokaryotic genes in eukaryotic cells and Reverse primer: 5'-AAGCTCGAGGAATTCTTAACGCAGTAAAGAGAGGAC-3' containing XhoI and EcoRI restriction sites with a translation stop codon.

PCR reaction was carried out with 30 cycles of denaturation at $94^{\circ} \mathrm{C}$ for 1 minute, $58^{\circ} \mathrm{C}$ for 1 minute, $72^{\circ} \mathrm{C}$ for 2 minutes. The reaction was initiated at $94^{\circ} \mathrm{C}$ for 5 minutes as initial denaturation before beginning the PCR cycle, and it was ended with a final extension at $72^{\circ} \mathrm{C}$ for $10 \mathrm{~min}$ utes in a thermal cycler (TECHNE, UK).Finally, the amplified DNA of $f l i C$ gene was visualized by electrophoresis on $1 \%$ agarose gel and $\mathrm{fliC}$ gene fragments were purified from the PCR product using a High Pure PCR Product Purification Kit (Roche, Germany).

\subsection{Gene Cloning}

Recovered fliC gene was cloned into pPrimecloning vector by Perfect PCR Cloning Kit (5prime), according to the manufacturer's instruments. Ligation reaction was prepared in a $10 \mu \mathrm{l}$ volume containing; $54 \mathrm{ng}$ of $\mathrm{fliC}$ gene, $50 \mathrm{ng}$ of pPrimecloning vector, $5 \mu \mathrm{l}$ of $2 \mathrm{X}$ ligation master mix and $2.5 \mu \mathrm{l}$ of distilled water. This reaction was incubated at $15^{\circ} \mathrm{C}$ for 16 hours. The ligation reaction was transformed to E. coli DH5 $\alpha$ strain competent cells and dispensed on agar plate containing $50 \mu \mathrm{g} / \mathrm{mL}$ of kanamycin (Sigma, USA), $200 \mathrm{mg} / \mathrm{mL}$ of Isopropyl $\beta$-D-1thiogalactopyranoside IPTG (Sigma, USA) and $20 \mathrm{mg} / \mathrm{mL}$ of X-Gal (Sigma, USA) to discriminate between recombinant (white) and non-recombinant (blue) cells. The plates were incubated at $37^{\circ} \mathrm{C}$ for 16 hours. A number of white colonies were assayed by colony PCR. After selecting the recombinant clones, the recombinant fliC plasmid was extracted from the overnight culture by High Pure Plasmid Isolation Kit (Roche, Germany) according to the manufacturer's protocol. The recombinant plasmids were detected by restriction digestion with NheI and XhoI enzymes (New England BioLabs, USA) and confirmed by sequence analysis (Bioneer, Korea).

\subsection{Subcloning in pVAX1 Eukaryotic Expression Vector}

The recombinant plasmid pPrime-fliC and eukaryotic 
Taherkhani R et al.

expression vector pVAX1 (Invitrogen, Carlsbad, CA, USA) were digested by XhoI and NheI enzymes (New England BioLabs, USA) at $37^{\circ} \mathrm{C}$ for 16 hours. After the enzymes thermal inactivation, the products of digestion were analyzed by electrophoresis on $1 \%$ agarose low melting point gel (Roche, Germany) and bands of fliC fragments and digested pVAX1 plasmids were purified from the agarose gel by the Agarose Gel DNA Extraction Kit (Roche, Germany).

The fliC gene was subcloned in the pVAX1 expression vector. Ligation reaction was prepared in a $20 \mu$ volume containing; $300 \mathrm{ng}$ of fliC gene, $200 \mathrm{ng}$ of digested pVAX1, $2 \mu \mathrm{l}$ of $10 \mathrm{X}$ ligation buffer and $1 \mu \mathrm{l}$ of T4 DNA ligase enzymes (New England BioLabs, USA). This reaction was incubated at $15^{\circ} \mathrm{C}$ for 16 hours. The recombinant pVAX-fliC plasmid was transformed into competent E. coli $\mathrm{DH} 5 \alpha$ cells and recovered in super optimal broth with catabolite repression (SOC) free of antibiotic at $37^{\circ} \mathrm{C}$ for one hour. Next, the cells were cultured on LB agar medium containing $50 \mu \mathrm{g} / \mathrm{mL}$ of kanamycin (Sigma, USA), and incubated at $37^{\circ} \mathrm{C}$ for 16 hours. A number of colonies were assayed by colony PCR and recombinant plasmids with the correct restriction pattern were selected by restriction digestion with NheI and XhoI enzymes (New England BioLabs, USA) and used for DNA sequencing with the T7 Forward and bovine growth hormone (BGH)reverse primers (Bioneer, Korea) to confirm that fliC gene is cloned in the proper orientation.

\subsection{Expression of Recombinant Flagellin in Mam- malian Cells}

Hela cells and HEK293 cells were cultured in Dulbecco's Modified Eagle's medium (DMEM) (GIBCO-BRL) supplemented with $10 \%$ fetal calf serum (FCS) in the presence of penicillin $\mathrm{G}(100 \mathrm{U} / \mathrm{mL})$, streptomycin $(100 \mu \mathrm{g} / \mathrm{mL})$, gentamycin $(50 \mu \mathrm{g} / \mathrm{mL})$ and amphotericin $\mathrm{B}(2.5 \mu \mathrm{g} / \mathrm{mL})$ (GIBCO-BRL) at $37^{\circ} \mathrm{C}$ with $5 \% \mathrm{CO}_{2}$ and transiently transfected with either pVAX-fliC or pVAX1 by electroporation. Briefly, growing cells were trypsinized, spun down, and resuspended in HeBS buffer (25 mM Hepes $\mathrm{pH}$ 7.1, 140 $\mathrm{mMNaCl}, 5 \mathrm{mM} \mathrm{KC1}, 0.75 \mathrm{mM} \mathrm{Na}_{2} \mathrm{HPO}_{4}$ ) at cell concentration of $5 \times 106 / \mathrm{mL}$ containing $100 \mu \mathrm{g} / \mathrm{mL}$ plasmid, and electroporated at room temperature in a $2 \mathrm{~mm}$ gap cuvette with $200 \mu \mathrm{L}$ of sample, exponential decay waveform of $160 \mathrm{~V}$ pulse and $500 \mu \mathrm{F}$ capacitance by Gene PulserXcell electroporation system (BIORAD-USA).

Electroporated cells were cultured in six-well culture plates (Nunc, Denmark) at $37^{\circ} \mathrm{C}$ in a $5 \% \mathrm{CO}_{2}$ environment. Forty-eight hours after transfection, the cells were fixed with cold methanol-acetone (1:1) for 20 minutes, then rinsed and blocked with $2 \mathrm{~mL}$ of $0.15 \mathrm{M}$ phosphate buffered saline (PBS) containing $1 \%$ bovine serum albumin (BSA). Flagellin expression was detected using the indirect immunofluorescence assay (IFA), with $2.5 \mu \mathrm{g} / \mathrm{mL}$ mouse anti-fliC (flagellin) antibody (Biolegend, USA) followed by staining with $10 \mu \mathrm{g} / \mathrm{mL}$ fluorescein isothiocya- nate (FITC)-labeled anti-mouse IgG (Abcam, USA). Next, 1 $\mu \mathrm{g} / \mathrm{mL}$ 4',6-diamidine-2'-phenylindole dihydrochloride (DAPI) (Roche, Germany) was used for staining the nuclei of the cells. After rinsing with PBS, cover slips were immediately observed under a fluorescence microscope (Nikon, USA).

The expression of flagellin in electroporated $\mathrm{CHO}$ cells and HEK293 cells was identified by western blotting. The transfected cells were maintained in DMEM-F12 (GIBCO$\mathrm{BRL}$ ) at $37^{\circ} \mathrm{C}$ and $5 \% \mathrm{CO} 2$ atmosphere. After 48 hours of incubation, the cells were collected and boiled in the sodium dodecyl sulfate (SDS) sample buffer (60 mMTris$\mathrm{HCl}$ pH 6.8, 2\% SDS, 1 mM Ethylene diaminetetraacetic acid (EDTA), 10\% glycerol, $0.005 \%$ bromophenol blue and $5 \% \beta$-mercaptoethanol) for 10 minutes. Proteins of transfected cells were separated using 10\% (w/v) SDS-PAGE gel. Following electrophoresis, the resolved proteins were blotted onto a $0.45 \mu \mathrm{m}$ pore polyvinylidene fluoride (PVDF) membrane (Roche, Germany) in towbin buffer (0.025 M tris, $0.192 \mathrm{M}$ glycine ( $\mathrm{pH}=8.3$ ) with $20 \%$ methanol) using a semi-dry transfer cell (Bio-Rad, USA) at $15 \mathrm{~V}$ for 1 hour and the membrane was blocked for 1 hour at room temperature with $3 \%(\mathrm{w} / \mathrm{v})$ BSA diluted in tris-buffered saline (TBS) (50 Mm tris $\mathrm{pH}=7.5,150 \mathrm{mM} \mathrm{NaCl}$ ). The membrane was incubated with $1 \mu \mathrm{g} / \mathrm{mL}$ mouse anti-fliC (flagellin) antibody (Biolegend, USA) overnight at $4{ }^{\circ} \mathrm{C}$. After washing with TBS containing $0.1 \%$ tween 20 , the membrane was stained with diluted goat anti-mouse IgG horseradish peroxidase (HRP)-labeled secondary antibody (1:2,000; Biolegend, USA) for 1 hour. The reaction was detected using 3, 3'-Diaminobenzidine (DAB) reagents (Sigma-Alderich, USA).

Transcription of flagellin gene at the level of mRNA in $\mathrm{CHO}$ and Hela cells was also identified by RT-PCR. Total RNA isolation from the cells by RNeasy Mini Kit (Qiagen, Germany) and RT-PCR was performed according to the manufacturer's protocol using a QIAGEN One Step RT-PCR Kit (Qiagen, Germany) by specific primers (forward primer: 5'-CGTATCAACAGCGCGAAAGA-3' and reverse primer: 5'-CTGGATGGAGTCGAGGTCAG-3'); the final product was then electrophoresed on $2 \%$ agarose gel.

\section{Results}

Genomic DNA of S. enterica serovar typhimurium extracted using a commercial kit showed a concentration of $170 \mu \mathrm{g} / \mathrm{mL}$ and good quality on agarose gel. The full protein-coding region of the fliC gene of S. typhimurium was amplified using designed specific primers. The size of PCR product on agarose gel electrophoresis for fliC gene was $1.5 \mathrm{kbp}$ (Figure 1). The PCR products were cloned into a pPrime cloning vector and this resulted in a recombinant pPrime-fliC plasmid that was transformed successfully into E. coli bacteria. The recombinant plasmids from the white colonies were PCR positive against the fliC gene and vector primers. The recombinant pPrime-fliC plasmids were confirmed by double diges- 
Taherkhani R et al.

tion with NheI and XhoI restriction enzymes (Figure 2). Sequencing analysis of the constructed plasmid also confirmed no amplification errors in sequence of the cloned flic gene.

Next, this pPrime-fliC plasmid was digested by NheI and XhoI restriction enzymes and $\mathrm{fliC}$ gene was successfully inserted between the NheI and XhoI restriction sites of the pVAX1 expressing eukaryote vector and the pVAX-fliC plasmids were transformed into E. coli $\mathrm{DH} 5 \alpha$ cells. The recombinant pVAX-fliC plasmids were detected by digestion with NheI and XhoI restriction enzymes (Figure 3) and were then confirmed by sequence analysis and compared with fliC sequences in the GenBank database. The $f l i C$ gene sequence was $100 \%$ identical to S. enterica subsp. enteric serovar typhimurium ATCC 14028, accession no.: NC_016856. In order to examine the in vitro expression of the flagellin protein, eukaryotic expression vector pVAX-fliC was electroporated successfully into Hela cells, HEK293 cells and CHO cells. Transcription of fliC mRNAs was confirmed by RT-PCR method; finally the results were electrophoresed on $2 \%$ agarose gel, which identified a band 231 bp (Figure 4). Expression of flagellin protein in Hela cells and HEK293 cells was evaluated by IFA, 48hours post-electroporation (Figure 5). Also, expression of the flagellin protein was evaluated by western blotting with cell lysates of transient transfected CHO cells and HEK293 cells. The molecular weight of the flagellin protein was about $52 \mathrm{kDa}$ (Figure 6).

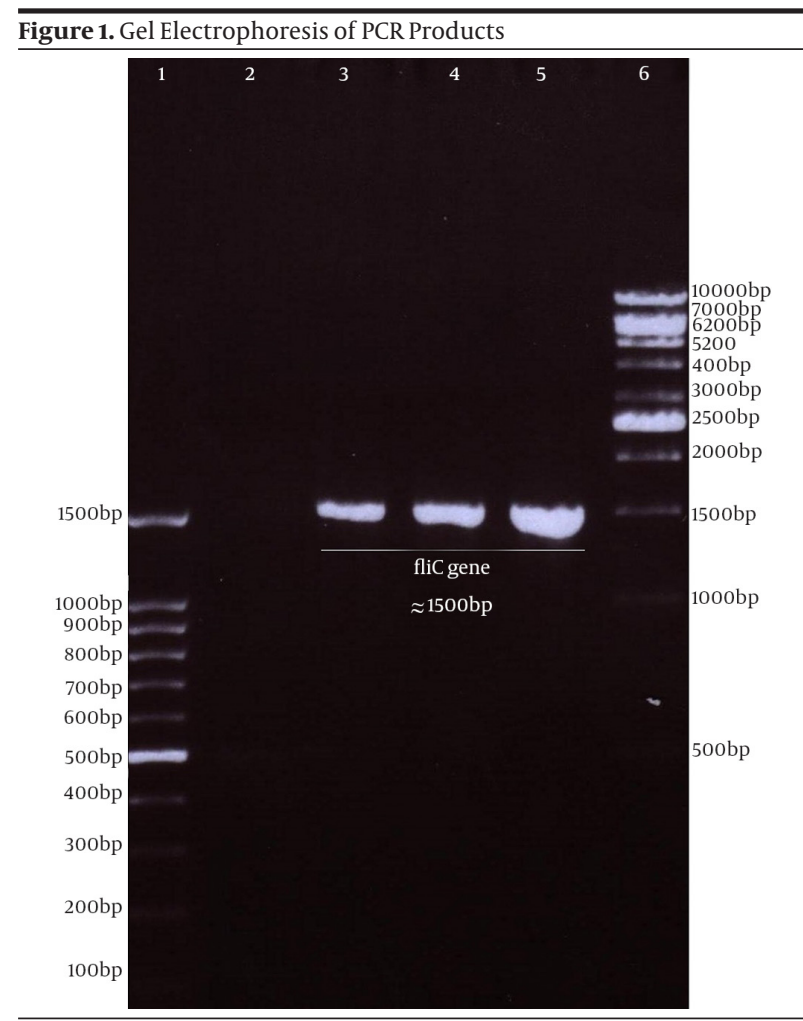

Lane 1:100bp DNA ladder Cinagen; Lane 2: negative control; Lane 3, 4 and 5: PCR products for flic gene (approximately 1500bp) of Salmonella enterica serovar typhimurium; Lane 6:1Kbp DNA ladder vivantis.

Figure 2. Electrophoresis of pPrime-fliC Recombinant Plasmids From White Colonies on 1\% Agarose Gel

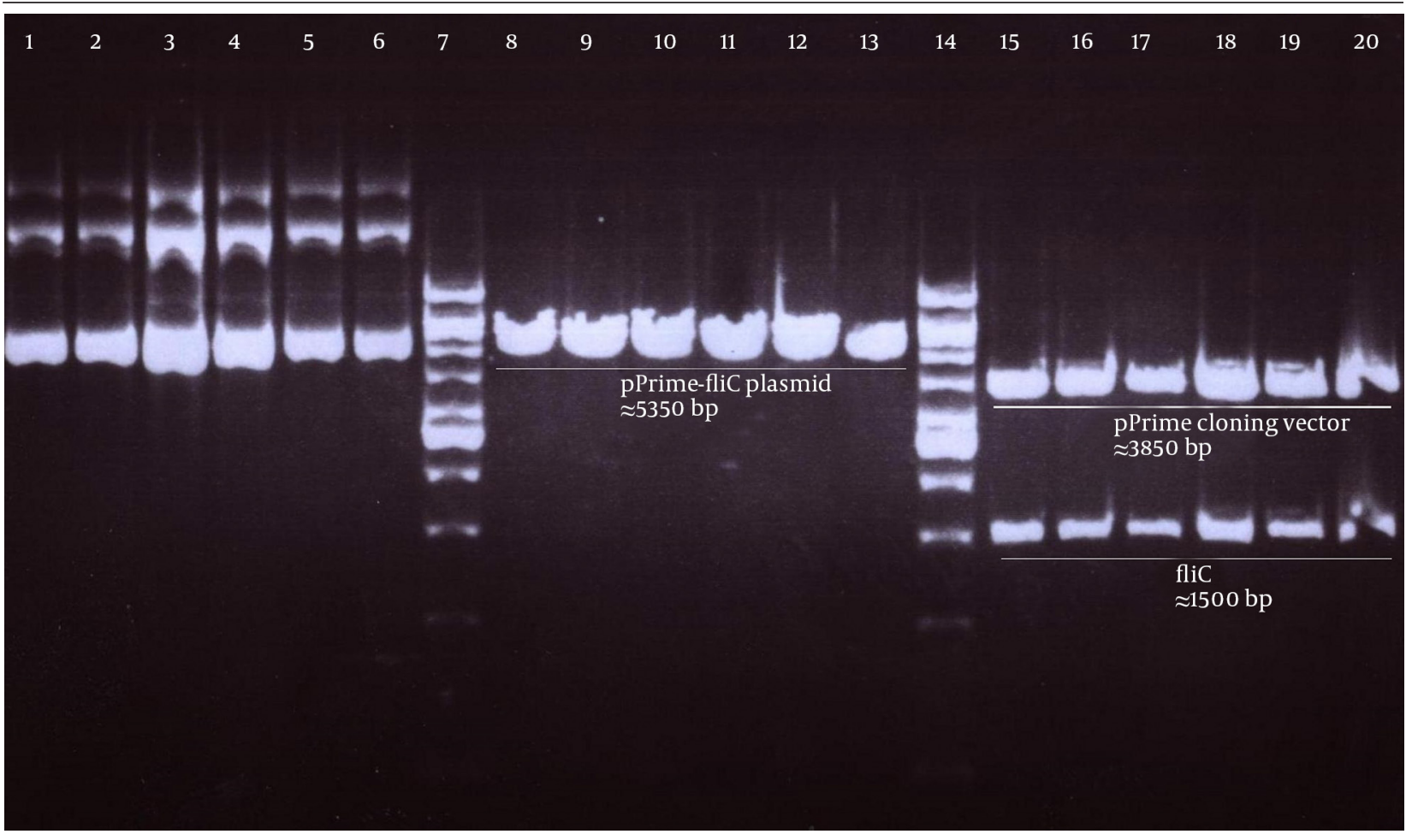

Lane 1 to 6: extracted Pprime-fliC plasmids; Lane 8 to 13: Pprime-fliC plasmids digested by XhoI; Lane 15 to 20: Pprime-fliC plasmids digested by XhoI and NheI; Lane 7 and 14:1 Kbp DNA ladder vivantis. 
Taherkhani R et al.

Figure 3. AgaroseGel Electrophoresis of Restriction Enzyme Digestion of Purified pVAX-fliC Recombinant Plasmid

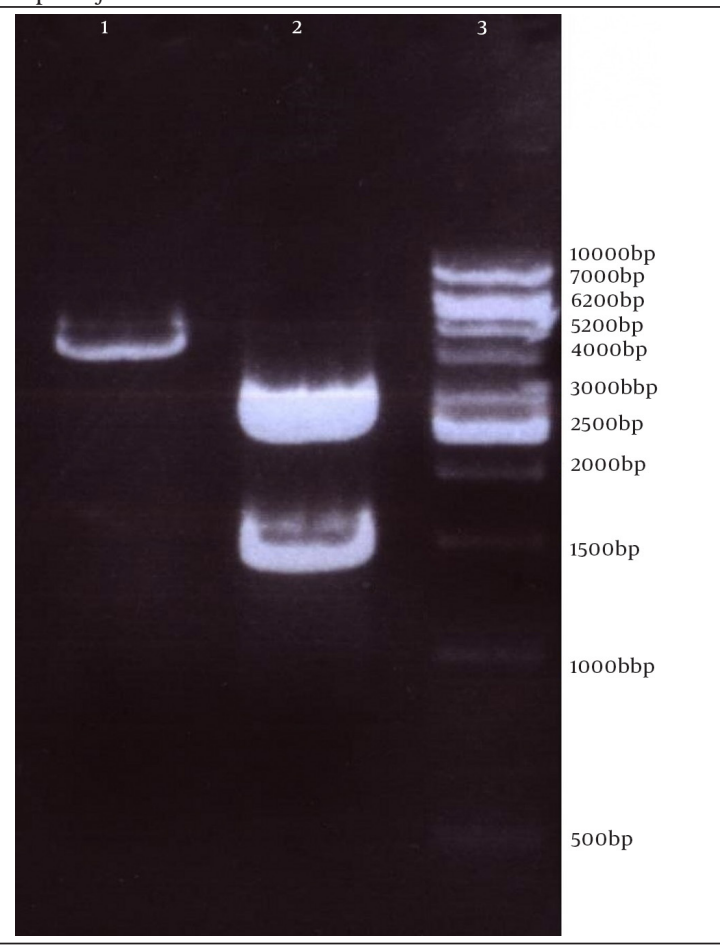

Lane 1: pVAX-fliC plasmid digested by XhoI had one band (4419 bp); Lane 2: Double Digestion by NheI and XhoI on pVAX-flic plasmid had two bands that were 1509 bp (down) and 2910 bp (up); Lane 3: 1 Kbp DNA ladder Vivantis.

\section{Figure 4. RT-PCR Amplification of the fliC Gene From Transfected Cells} and Electrophoresis on $2 \%$ Agarose Gel

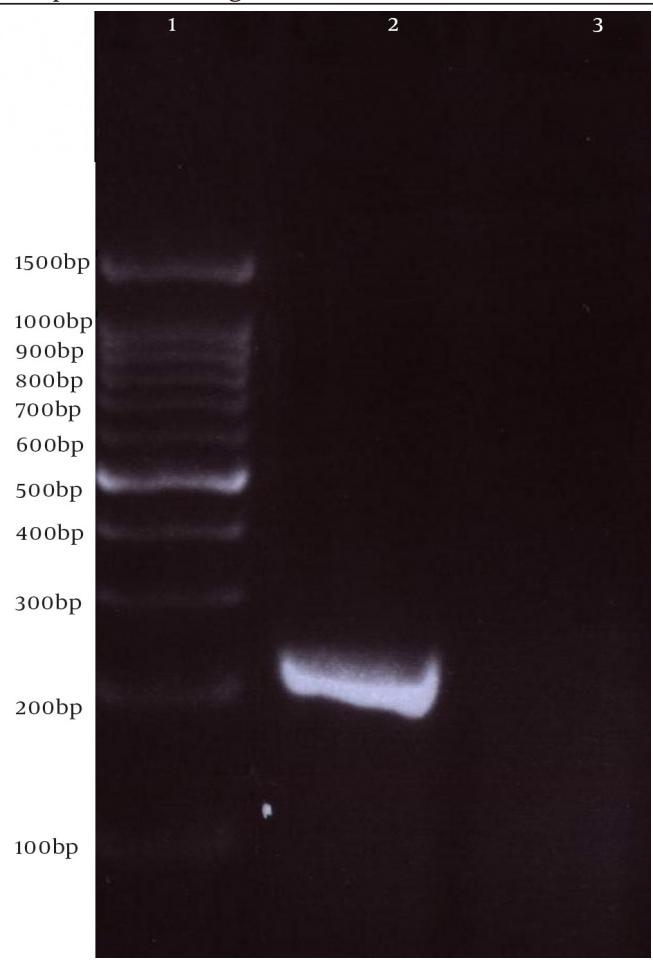

Lane 1:100 bp DNA ladder Cinnagen Co. Lane 2: RT-PCR product of the fliC gene (231 bp) from transfected cells with pVAX-fliC plasmid; Lane 3: RT-PCR Product of the fliC gene from transfected cells with pVAX plasmid (negative control).

Jundishapur J Microbiol. 2014;7(11):e12351
Figure 5. Detection of Flagellin Protein on Hela cells (A) and HEK293 Cells (B) by Indirect Fluorescent Antibody Test
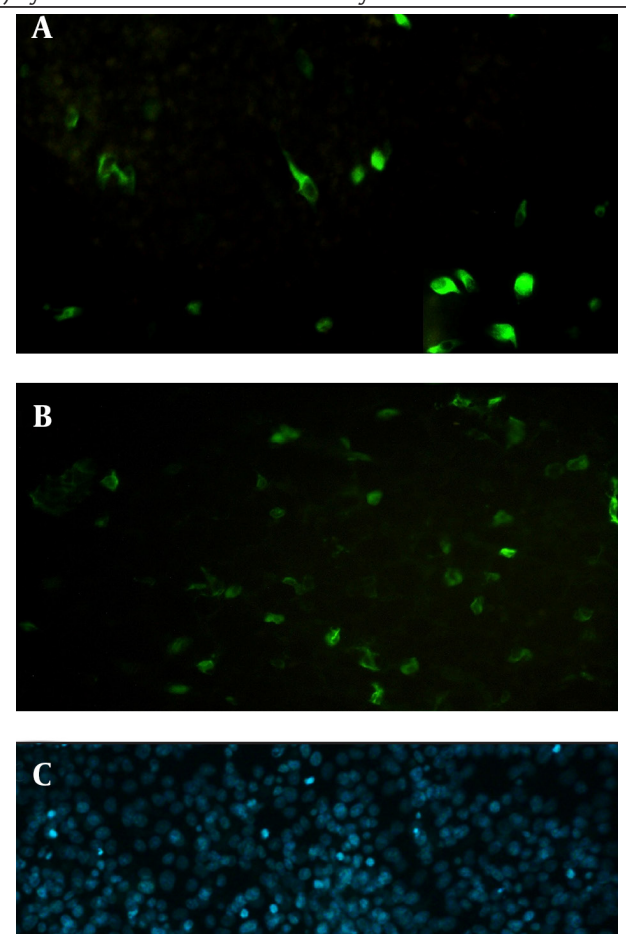

The cells were previously electroporated with pVAX-fliC and detected using mouse anti-fliC antibody and FITC-labeled goat anti-mouse IgG antibody. DAPI was used for staining the nuclei of Hela cells (C).

Figure 6. Expression Analysis of Flagellin Protein in Transfected Chinese Hamster Ovary Cells by Western Blotting

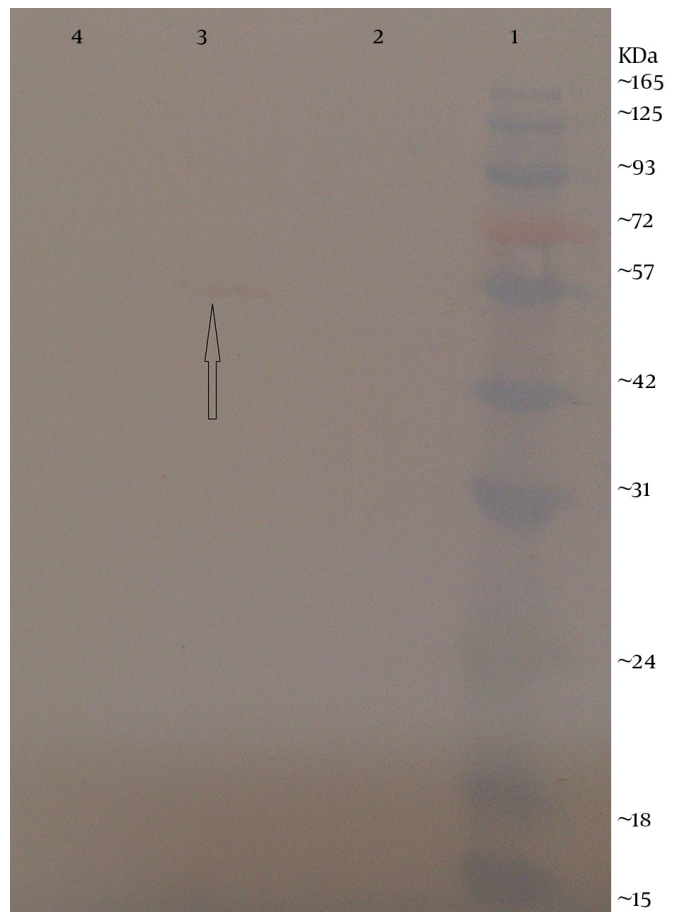

Untransfected (lane 2) and transfected with pVAX1-fliC (lane 3) or transfected with pVAX1 (lane 4) CHO cells were detected by western blotting Lane 1, prestained protein ladder(Cinagen, Iran). The molecular weight of flagellin protein was about $52 \mathrm{kDa}$ 


\section{Discussion}

Production of a strong immune response and induction of both humoral and cellular immunity against vaccine antigens strongly depend on a formulation with adjuvants. Immunologic adjuvants are agents incorporated into vaccine formulations to enhance the immunogenicity of vaccine antigens (19). Many natural and synthetic compounds as immunological adjuvants including inorganic salts such as aluminum hydroxide, nano and micro particles, oil emulsions, cytokines and microbial compounds, have been studied in vaccine researches (19, 20 ). The use of microbial-adjuvants in human prophylactic vaccines which can induce the production of endogenous cytokines have priority over the use of recombinant cytokines as adjuvants that have high incidence of local and systemic adverse side effects (21).

A primary mechanism of action for most microbialbased immunological adjuvants is to present pathogenspecific molecular patterns (PAMPs) in association with protective antigens triggering pathogen recognition mechanisms and the activation of innate immunity. The recognition of PAMPs in mammalian cells is mediated by innate immune receptors such as pattern recognition receptors (PRRs), which are expressed by antigen-presenting cells (APC). However, activation of the PRRs such as TLRs not only induces strong inflammatory responses but also stimulates protective adaptive immune responses (3). Recent advances in the field of innate immune system have disclosed cellular and molecular mechanisms behind the adjuvant effects of PAMPs and have led to the use of TLR ligands as adjuvants $(1,6)$. TLRs are a type of PRR which react with PAMP-based adjuvants or microbial adjuvants. Therefore, the use of TLR agonists such as flagellin (a bioactive TLR5 ligand) as adjuvants is a promising approach to enhance the effectiveness of vaccines and may be the most useful for vaccines that may lack sufficient recognition by host innate immune mechanisms.

Flagellin, a TLR5 ligand, is the major protein component of bacterial flagella, which provide motility and chemotaxis, and allow adhesion to host mucosal tissues (22). The TLR5 ligand is a potent T-cell antigen and has potential as a vaccine adjuvant. Unlike other TLR agonists, flagellin tends to produce mixed Th1 and Th2 responses rather than strong Th1 responses. Additionally, in contrast to other vaccine adjuvants, such as complete Freund's adjuvant, flagellin may exert strong adjuvant effects on both humoral and cellular immune responses following administration through mucosal routes (12). Flagellin can be used as an adjuvant mixed with the antigen but it is more frequently fused to a recombinant vaccine antigen $(5,11,12)$. In addition to the upregulation of TLR5 expression, TLR3 expression was also found to be activated by flagellin, thus it could be auseful against for RNA viruses (16). Flagellin is a strong activator of a broad range of cell types involved in innate and adaptive immunity. Moreover, Delaney et al. established that flagellin can promote robust responses against weakly immunogenic antigens of vaccinia virus (23). The efficacy of preventive vaccines against infectious diseases declines dramatically with the progression of age among older adults (24). Adjuvants which lead to an increase in immunogenicity, are required to stimulate the appropriate immunity in the elderly. Bates et al. indicated that flagellin can promote adaptive immune responses in aged mice (13).

According to the study of Vijay-Kumar et al., systemic treatment of purified flagellin in mice did not induce serological, histopathological and clinical hallmarks of inflammation that are induced by lipopolysaccharides, yet protected mice against chemicals, pathogens and ionizing radiation (18). In addition, bacterial genes typically contain immunostimulatory unmethylated CpG motifs (PuPuCpGPyPy) within their DNA backbone (25). We Investigated the $\mathrm{CpG}$ motifs in the entire sequence of the fliC gene and observed that the $\mathrm{fliC}$ gene's sequence contains four immunostimulatory CPG motif sites, including agcgtc at positions 89-94, aacgct at positions 199-204, agcgcc at positions 353-358 and aacgtc at positions 14651470 (based on GenBank accession number NC_016856, Region: 2059063-2060550).

The aim of the present study was to construct the expression plasmid pVAX-flic and evaluate its expression in eukaryotic cells. Initially, the full-length of fliC gene from S. typhimurium was amplified using specific primers containing the Kozak sequence and cloned in thepPrime vector and then sub-cloned in expression plasmid pVAX1. Next, cloning and subcloning was confirmed by PCR, restriction enzyme digestion and DNA sequencing. The DNA sequencing results also confirmed the authenticity of the cloned flagellin gene sequences. Following this step, mRNA synthesis of flagellin in eukaryotic cells transfected with pVAX-fliC was confirmed by the RT-PCR test. Also, the presence of flagellin protein was determined in these cells by immunofluorescence assay and western blotting using a monoclonal antibody. Better results were seen for the immunofluorescence assay with HEK293 cells compared to Hela cells. Therefore, it is better to use HEK293 cells to evaluate the expression plasmid. Western blot analysis showed a protein band slightly lower than the $57 \mathrm{kDa}$ band of the protein marker, which was in agreement with the expected molecular weight of flagellin protein ( $\approx 52 \mathrm{kDa}$ ). The results of this study indicated that the flagellin gene was successfully cloned into the expression plasmid pVAX1, and the expression of this gene at the level of transcription and translation was confirmed in eukaryotic cells. The construction of the pVAXfliC recombinant plasmid is the first step towards the use of this plasmid as a genetic adjuvant candidate in vaccine studies in the future.

Applequist et al. cloned the membrane-associated form of flagellin in expression vector pcDNA3.1 (26). However, in our study, the full-length fliC gene was cloned in the pVAX1 expression vector, and the sequence of the transmembrane domain or secretory signal sequence was not 
used. This is because the immune response to antigens that are on the cell surface may be different from the antigens, which are not on the cell surface and could play a role in the polarizing immune responses and raising different profiles of T-cells (27). The adjuvant effect of flagellin has been shown by a variety of pathogen models. An increasing number of studies have demonstrated that flagellin contributes to the efficacy of various novel vaccines for Yersinia pestis (15), West Nile virus (28), Plasmodium falciparum (29), Schistosoma mansoni (30), Tetanus toxoid (31) and influenza (17,32-34). Flagellin activates TLR5-positive dendritic cells (DCs), neutrophils and epithelial cells $(2,35)$. Therefore, a powerful vaccine can be created by fusion of flagellin with an antigen in order to enhance the immunopotency of the antigens (19). However, in most mentioned studies they used flagellin as a recombinant protein for vaccine adjuvants, however in this study, for the first time, we inserted fliC gene in pVAX1 for DNA vaccine as an adjuvant candidate.

In conclusion, the recombinant plasmid of pVAX-fliC that was successfully constructed in the present study could be used either for cocktail or physical linkage to target antigens as an effective DNA microbial-adjuvant for future DNA vaccine studies against infectious diseases.

\section{Acknowledgements}

The authors would like to acknowledge the financial support of the Infectious and Tropical Diseases Research Center of Ahvaz Jundishapur University of Medical Sciences, Ahvaz, IR Iran (grant No. 91112).

\section{Authors' contributions}

Manoochehr Makvandi takes responsibility for the accuracy of the data. Fatemeh Farshadpour and Reza Taherkhani contributed equally to the design and performance of the study. Ali Reza Samarbafzadeh participated in the laboratory evaluation. Manoochehr Makvandi drafted the manuscript and is the guarantor. All authors have read and approved the final manuscript.

\section{Funding/Support}

Financial support was provided by the Infectious and Tropical Diseases Research Center of Ahvaz Jundishapur University of Medical Sciences, Ahvaz, IR Iran (grant No. 91112).

\section{References}

1. Kaisho T, Akira S. Toll-like receptors as adjuvant receptors. Biochim Biophys Acta. 2002;1589(1):1-13.

2. Akira S, Uematsu S, Takeuchi O. Pathogen recognition and innate immunity. Cell. 2006;124(4):783-801.

3. Takeda K, Kaisho T, Akira S. Toll-like receptors. Annu Rev Immunol. 2003;21:335-76.

4. Pasare C, Medzhitov R. Toll-like receptors: linking innate and adaptive immunity. Microbes Infect. 2004;6(15):1382-7.

5. Huleatt JW, Jacobs AR, Tang J, Desai P, Kopp EB, Huang Y, et al.
Vaccination with recombinant fusion proteins incorporating Toll-like receptor ligands induces rapid cellular and humoral immunity. Vaccine. 2007;25(4):763-75.

6. Coffman RL, Sher A, Seder RA. Vaccine adjuvants: putting innate immunity to work. Immunity. 2010;33(4):492-503.

7. Gewirtz AT, Simon PO, Jr, Schmitt CK, Taylor LJ, Hagedorn CH, $\mathrm{O}$ Brien $\mathrm{AD}$, et al. Salmonella typhimurium translocates flagellin across intestinal epithelia, inducing a proinflammatory response. J Clin Invest. 2001;107(1):99-109.

8. Gewirtz AT. Flag in the crossroads: flagellin modulates innate and adaptive immunity. Curr Opin Gastroenterol. 2006;22(1):8-12.

9. Hayashi F, Smith KD, Ozinsky A, Hawn TR, Yi EC, Goodlett DR, et al. The innate immune response to bacterial flagellin is mediated by Toll-like receptor 5. Nature. 2001;410(6832):1099-103.

10. Gewirtz AT, Navas TA, Lyons S, Godowski PJ, Madara JL. Cutting edge: bacterial flagellin activates basolaterally expressed TLR5 to induce epithelial proinflammatory gene expression. J Immunol. 2001;167(4):1882-5.

11. Honko AN, Mizel SB. Effects of flagellin on innate and adaptive immunity. Immunol Res. 2005;33(1):83-101.

12. Mizel SB, Bates JT. Flagellin as an adjuvant: cellular mechanisms and potential.JImmunol.2010;185(10):5677-82.

13. Bates JT, Honko AN, Graff AH, Kock ND, Mizel SB. Mucosal adjuvant activity of flagellin in aged mice. Mech Ageing Dev. 2008;129(5):271-81.

14. Cuadros C, Lopez-Hernandez FJ, Dominguez AL, McClelland $\mathrm{M}$, Lustgarten J. Flagellin fusion proteins as adjuvants or vaccines induce specific immune responses. Infect Immun. 2004;72(5):2810-6.

15. Honko AN, Sriranganathan N, Lees CJ, Mizel SB. Flagellin is an effective adjuvant for immunization against lethal respiratory challenge with Yersinia pestis. Infect Immun. 2006;74(2):1113-20.

16. Chaung HC, Cheng LT, Hung LH, Tsai PC, Skountzou I, Wang B, et al. Salmonella flagellin enhances mucosal immunity of avian influenza vaccine in chickens. Vet Microbiol. 2012;157(1-2):69-77.

17. Huleatt JW, Nakaar V, Desai P, Huang Y, Hewitt D, Jacobs A, et al. Potent immunogenicity and efficacy of a universal influenza vaccine candidate comprising a recombinant fusion protein linking influenza M2e to the TLR5 ligand flagellin. Vaccine. 2008;26(2):201-14.

18. Vijay-Kumar M, Aitken JD, Sanders CJ, Frias A, Sloane VM, Xu J, et al. Flagellin Treatment Protects against Chemicals, Bacteria, Viruses, and Radiation. JImmunol. 2008;180(12):8280-5.

19. Wang W, Manmohan S. Selection of Adjuvants for Enhanced Vaccine Potency. WJ Vac. 2011;1:33-78.

20. Vogel FR, Hem SL. Immunological Adjuants. 5. Plotkin S, Orensteinn W, Offit P, Vaccines. editors. : Elsevier; 2008.

21. Hedlund J, Langer B, Konradsen HB, Ortqvist A. Negligible adjuvant effect for antibody responses and frequent adverse events associated with IL-12 treatment in humans vaccinated with pneumococcal polysaccharide. Vaccine. 2001;20(1-2):164-9.

22. Reichhart JM. TLR5 takes aim at bacterial propeller. Nat Immunol. 2003;4(12):1159-60.

23. Delaney KN, Phipps JP, Johnson JB, Mizel SB. A recombinant flagellin-poxvirus fusion protein vaccine elicits complement-dependent protection against respiratory challenge with vaccinia virus in mice. Viral Immunol. 2010;23(2):201-10.

24. Aw D, Silva AB, Palmer DB. Immunosenescence: emerging challenges for an ageing population. Immunology. 2007;120(4):43546.

25. Krieg AM. CPG motifs in bacterial DNA and their immune effects. Annu Rev Immunol. 2002;20:709-60.

26. Applequist SE, Rollman E, Wareing MD, Liden M, Rozell B, Hinkula J, et al. Activation of innate immunity, inflammation, and potentiation of DNA vaccination through mammalian expression of the TLR5 agonist flagellin.J Immunol. 2005;175(6):3882-91.

27. Sallberg M, Townsend K, Chen M, O'Dea J, Banks T, Jolly DJ, et al. Characterization of humoral and CD4+ cellular responses after genetic immunization with retroviral vectors expressing different forms of the hepatitis B virus core and e antigens.J Virol. 1997;71(7):5295-303.

28. McDonald WF, Huleatt JW, Foellmer HG, Hewitt D, Tang J, De- 
sai $\mathrm{P}$, et al. A West Nile virus recombinant protein vaccine that coactivates innate and adaptive immunity. J Infect Dis. 2007;195(11):1607-17.

29. Bargieri DY, Rosa DS, Braga CJ, Carvalho BO, Costa FT, Espindola $\mathrm{NM}$, et al. New malaria vaccine candidates based on the Plasmodium vivax Merozoite Surface Protein-1 and the TLR-5 agonist Salmonella Typhimurium FliC flagellin. Vaccine. 2008;26(48):613242.

30. Ben-Yedidia T, Marcus H, Reisner Y, Arnon R. Intranasal administration of peptide vaccine protects human/mouse radiation chimera from influenza infection. Int Immunol. 1999;11(7):1043-51.

31. Lee SE, Kim SY, Jeong BC, Kim YR, Bae SJ, Ahn OS, et al. A bacterial flagellin, Vibrio vulnificus FlaB, has a strong mucosal adjuvant activity to induce protective immunity. Infect Immun.
2006;74(1):694-702.

32. Ben-Yedidia T, Arnon R. Epitope-based vaccine against influenza Expert Rev Vaccines. 2007;6(6):939-48.

33. Adar Y, Singer Y, Levi R, Tzehoval E, Perk S, Banet-Noach C, et al A universal epitope-based influenza vaccine and its efficacy against H5N1. Vaccine. 2009;27(15):2099-107.

34. Turley CB, Rupp RE, Johnson C, Taylor DN, Wolfson J, Tussey L, et al. Safety and immunogenicity of a recombinant M2e-flagellin influenza vaccine (STF2.4xM2e) in healthy adults. Vaccine. 2011;29(32):5145-52.

35. Pino O, Martin M, Michalek SM. Cellular mechanisms of the adjuvant activity of the flagellin component FljB of Salmonella enterica Serovar Typhimurium to potentiate mucosal and systemic responses. Infect Immun. 2005;73(10):6763-70. 